\title{
Predictive Analysis for the Detection of Diabetes Mellitus (DM) based on Machine Learning Classification Algorithm
}

\author{
Dillip Narayan Sahu1, Vijay Pal Singh"* \\ ${ }^{1}$ Lecturer, Department of MCA, School of Computer Science, Gangadhar Meher University (GMU), Odisha, India \\ ${ }^{2}$ Associate Professor, Department of Computer Science, OPJS University, Rajasthan, India \\ *Corresponding Author: Dr. Vijay Pal Singh
}

\begin{abstract}
According to the World Health Organization, around 1.5 million people worldwide died due to diabetes in 2019. It is estimated that approximately 462 million people live with diabetes around the globe. According to other sources, about 432 million people worldwide have diabetes, the bulk living in low-and middle-income countries, and 1.5 million deaths are directly related to the disease diabetes annually. The amount of cases, morbidity and mortality rates in a specific time period or over time to time, the diabetes is steadily increasing over the past few decades. No doubt, Diabetes mellitus is a leading cause of deaths world wide and reduced life expectancy. This disease can be curable with early diagnosis and proper treatment. The purpose of this paper is to establish some predictive models using Machine Learning algorithms by taking a real time Diabetes mellitus dataset. In this paper, we have shown some real-time experiments and observations with the help of some Machine Learning algorithms, and also shown a clear picture on the predictive analysis for the detection of the disease Diabetes mellitus in medical science using Machine Learning algorithms using which patients may get accurate data so as to diagnose better for their early treatment.
\end{abstract}

Keywords: Algorithm, Classifier, Diabetes Mellitus, Machine Learning, Prediction.

\section{I.INTRODUCTION}

Machine learning tools are being extensively utilized in all scientific, medical fields and are liable for revolutionizing businesses everywhere. Healthcare systems, on the opposite hand, are very slow in adopting these advancements and are lagging far behind in this[1][2].

Machine learning are often useful within the management of chronic diseases, namely, diabetes[3][4]. In fact, Machine learning is already getting used to predict risk of diabetes supported genomic data, diagnosis of diabetes supported EHR data, to predict risk of complications. Adoption of Machine Learning technologies can significantly increase detection and early treatment of diabetic complications of patients[5][6].

\section{II.EXPERIMENTS AND OBSERVATIONS}

Here we have taken Diabetes dataset and Weka knowledge analysis tool to classify and predict the disease. The figure 1 and figure 2 shows the diabetes dataset and data preprocess respectively.

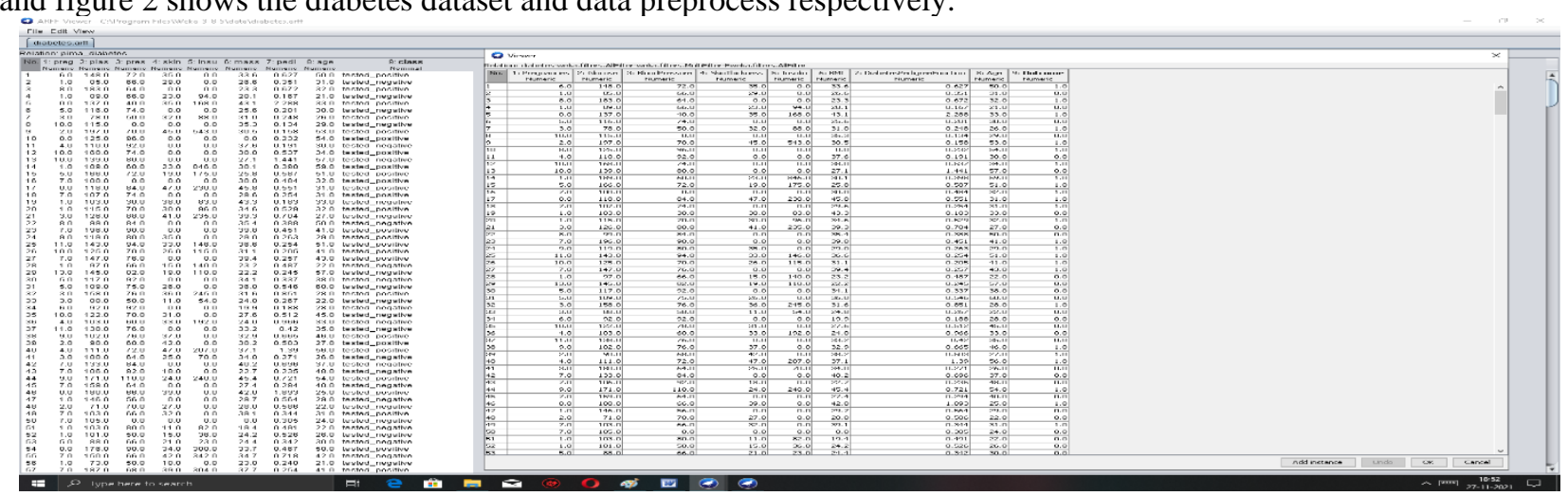

Fig.1. Diabetes Mellitus (DM) Dataset 


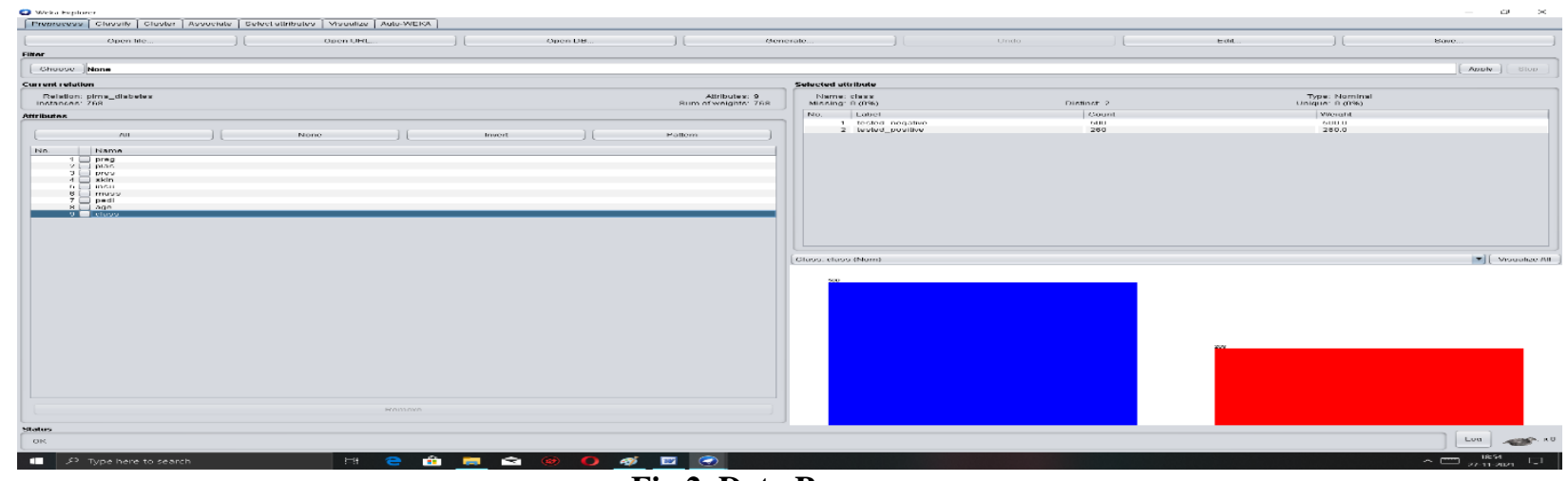

Fig.2. Data Preprocess

Experiments and Observations-1

Classifier Output=== Run information $===$

Scheme: weka.classifiers.rules.ZeroR

Relation: pima_diabetes

Instances: 768 Attributes: 9

Preg plas pres skin insu mass pedi age class

Test mode: 10-fold cross-validation

$===$ Classifier model (full training set) $===$

ZeroR predicts class value: tested_negative

Time taken to build model: 0 seconds

$===$ Stratified cross-validation $======$ Summary $===$

Correctly Classified Instances $\quad 500 \quad 65.1042 \%$

Incorrectly Classified Instances $268 \quad 34.8958 \%$

Kappa statistic

0

Mean absolute error

Root mean squared error

Relative absolute error

0.4766
$100 \%$
$100 \%$
768

$\begin{array}{ll}\text { Root relative squared error } & \\ \text { Total Number of Instances } & 768\end{array}$

=== Detailed Accuracy By Class ===

TP Rate FP Rate Precision Recall F-Measure MCC ROC Area PRC Area Class

$\begin{array}{llllllllll}1.000 & 1.000 & 0.651 & 1.000 & 0.789 & ? & 0.497 & 0.650 & \text { tested_negative } \\ 0.000 & 0.000 & ? & 0.000 & ? & ? & 0.497 & 0.348 & \text { tested_positive }\end{array}$

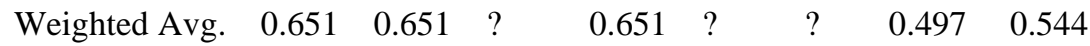

$===$ Confusion Matrix $===$

a $b \quad<--$ classified as

$5000 \mid \mathrm{a}=$ tested_negative

$2680 \mid \mathrm{b}=$ tested_positive

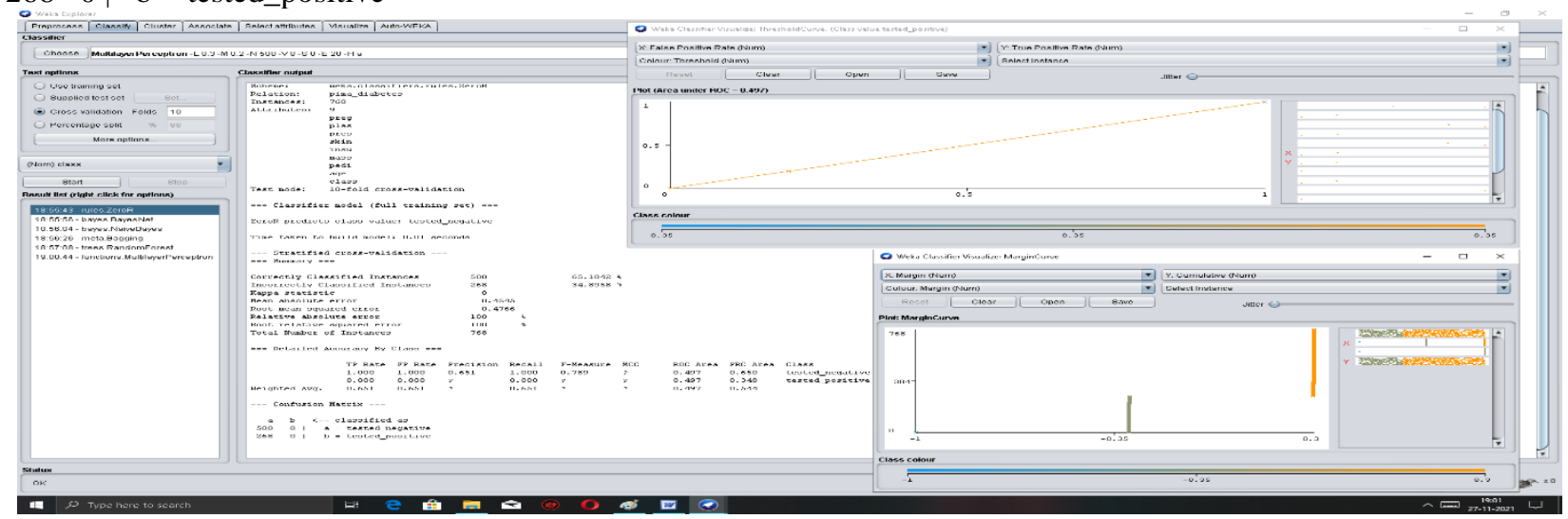

Fig.3. ZeroR Classifier with Visualize curve 


\section{Experiments and Observations-2}

Classifier Output=== Run information $===$

Scheme: weka.classifiers.bayes.BayesNet -D -Q weka.classifiers.bayes.net.search.local.K2 -- -P 1 -S BAYES -E weka.classifiers.bayes.net.estimate.SimpleEstimator -- -A 0.5

Relation: pima_diabetes Instances: 768

Attributes: 9

Test mode: 10-fold cross-validation

$===$ Summary $===$

Correctly Classified Instances $\quad 571 \quad 74.349 \%$

Incorrectly Classified Instances 197

Kappa statistic

Mean absolute error

Root mean squared error

Relative absolute error

Root relative squared error

Total Number of Instances

$===$ Confusion Matrix $===$
0.429

0.2987

0.4208

$65.7116 \%$

$88.28 \%$

768

a $b$ <-- classified as

40892 a $\mathrm{a}=$ tested_negative

$105163 \mid \mathrm{b}=$ tested_positive

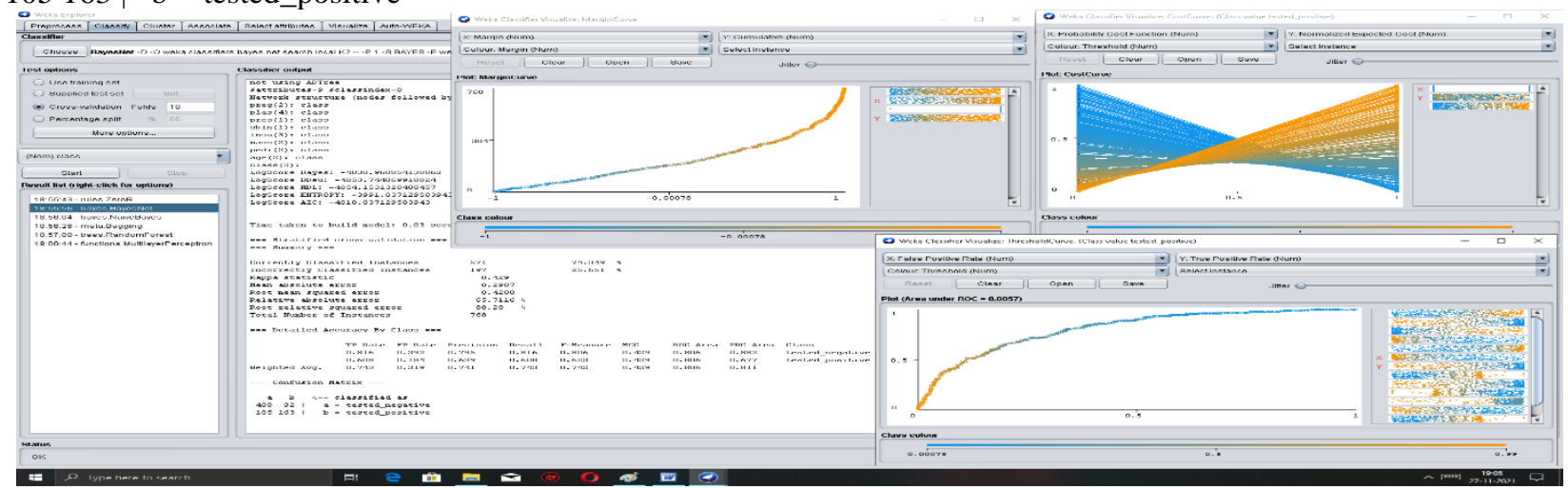

Fig.4. BayesNet Classifier with Visualize different cases

Experiments and Observations-3

Classifier Output $===$ Run information $===$

Scheme: weka.classifiers.bayes.NaiveBayesMultinomial

$===$ Classifier model (full training set) $===$

The independent probability of a class

tested_negative 0.65

tested_positive 0.35

$===$ Summary $===$

Correctly Classified Instances $\quad 460 \quad 59.8958 \%$

Incorrectly Classified Instances $308 \quad 40.1042 \%$

Kappa statistic $\quad 0.1279$

Mean absolute error $\quad 0.4009$

Root mean squared error $\quad 0.6152$

Relative absolute error $\quad 88.2029 \%$

Root relative squared error $\quad 129.0792 \%$

Total Number of Instances $\quad 768$

$===$ Confusion Matrix $===$

a $b \quad<--$ classified as

$339161 \mid \mathrm{a}=$ tested_negative

$147121 \mid \mathrm{b}=$ tested_positive 


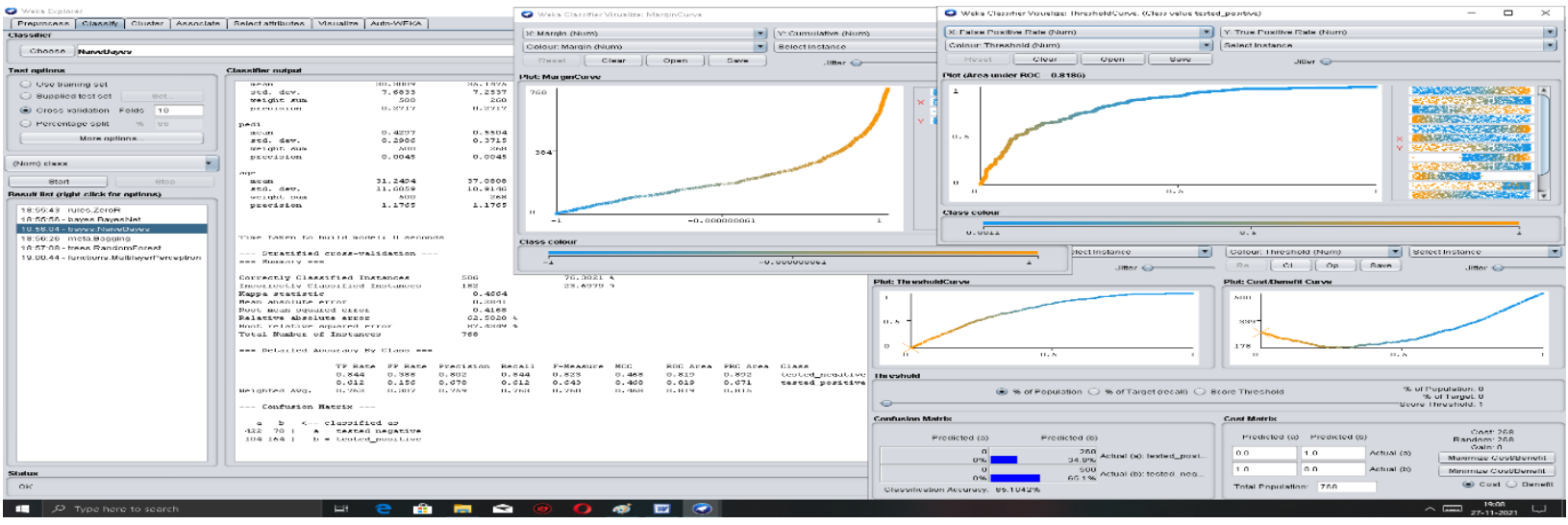

Fig.4. NaiveBayes Classifier with Visualize different cases

\section{Experiments and Observations-4}

Classifier Output $===$ Run information $===$

Scheme: $\quad$ weka.classifiers.meta.Bagging -P 100 -S 1 -num-slots 1 -I 10 -W weka.classifiers.trees.REPTree -- -M 2 -V 0.001 -N 3 -S 1 -L - 1 -I 0.0

Bagging with 10 iterations and base learner weka.classifiers.trees.REPTree -M 2 -V 0.001 -N 3 -S 1 -L -1 -I 0.0

$===$ Summary $==$

Correctly Classified Instances $\quad 582 \quad 75.7813 \%$

Incorrectly Classified Instances $186 \quad 24.2188 \%$

Kappa statistic $\quad 0.4498$

Mean absolute error $\quad 0.315$

Root mean squared error $\quad 0.4063$

Relative absolute error $\quad 69.3049 \%$

Root relative squared error $\quad 85.2474 \%$

Total Number of Instances $\quad 768$

$===$ Confusion Matrix $===$

$a \quad b \quad--$ classified as

$42575 \mid \mathrm{a}=$ tested_negative

$111157 \mid \mathrm{b}=$ tested_positive

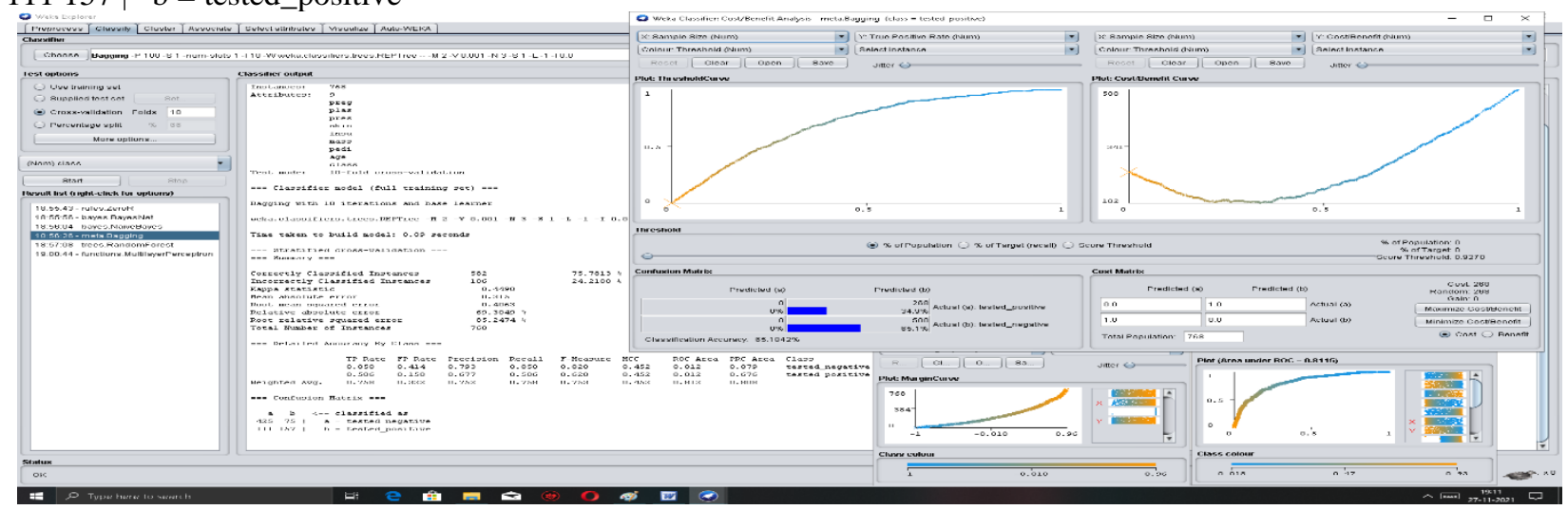

Fig.5. Bagging Classifier with Visualize different cases

Experiments and Observations-5

Classifier Output $===$ Run information $===$

Scheme: $\quad$ weka.classifiers.trees.RandomForest -P 100 -I 100 -num-slots 1 -K 0 -M 1.0 -V 0.001 -S 1 RandomForest weka.classifiers.trees.RandomTree -K 0 -M 1.0 -V 0.001 -S 1 -do-not-check-capabilities

$===$ Summary $===$

Correctly Classified Instances $\quad 582 \quad 75.7813 \%$

Incorrectly Classified Instances $186 \quad 24.2188 \%$

Kappa statistic $\quad 0.4566$

Mean absolute error $\quad 0.3106$ 
Root mean squared error Relative absolute error Root relative squared error Total Number of Instances $===$ Confusion Matrix $===$ a $b$ <-- classified as 41882 | a = tested_negative
0.4031

$68.3405 \%$

$84.5604 \%$ 768

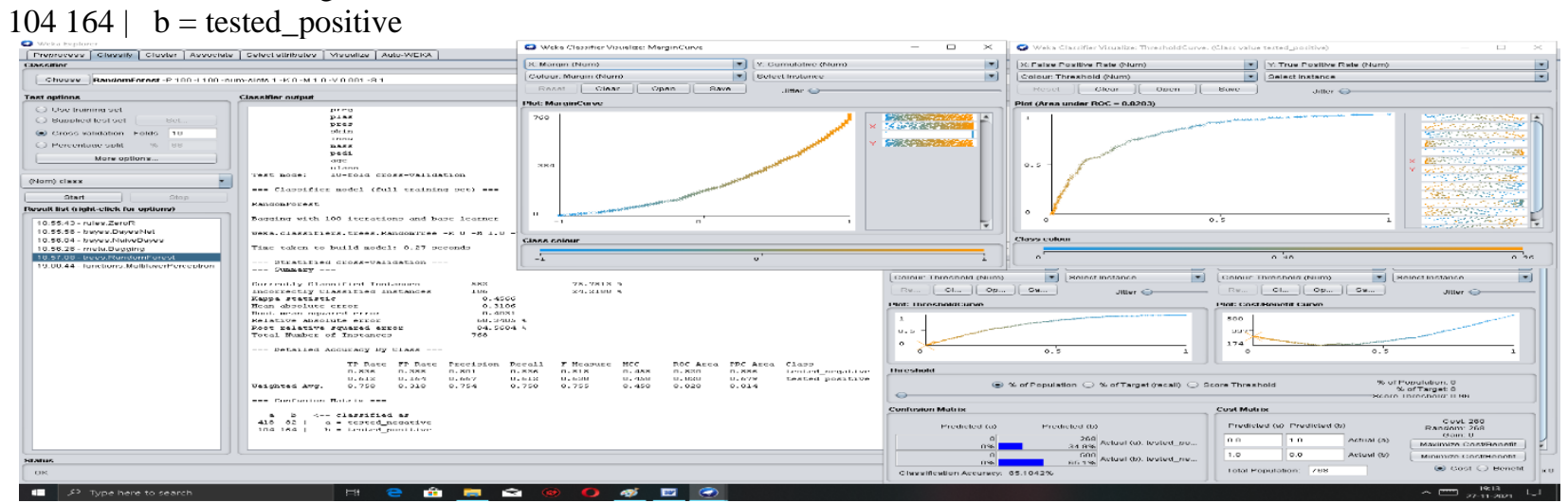

Fig.6. RandomForest Classifier with Visualize different cases

\section{III.DISCUSSION}

We have taken 5 different experimental observations using the machine learning tool to clearly analyze, detect and predict for the Diabetes Disease. In the study of the above experimental observations, it is found that, machine learning tools are no doubt an excellent way to predict and detect the Diabetes disease at an early stage prior to the satisfiability of the conditions of the early stage patient. It is found that the accuracy level using different algorithm in Machine Learning is an excellent option for detection and prediction of Diabetes disease, having good accuracy rate and so will be efficient and acceptable.

\section{IV.CONCLUSION}

In 2019, diabetes was the ninth leading cause of death with an estimated 1.5 million deaths directly caused by diabetes. Diabetes mellitus is a disease, which can cause many complications. How to exactly predict and diagnose this disease by using machine learning is worthy studying. According to the all above experiments, we found good accuracy using Random Forest and Bagging classifier so that will be acceptable.

\section{REFERENCES}

[1] Alghamdi, M., Al-Mallah, M., Keteyian, S., Brawner, C., Ehrman, J., and Sakr, S. (2017). Predicting diabetes mellitus using SMOTE and ensemble machine learning approach: the henry ford exercise testing (FIT) project. PLoS One 12:e0179805. doi: 10.1371/journal.pone.0179805

[2] American Diabetes Association (2012). Diagnosis and classification of diabetes mellitus. Diabetes Care 35(Suppl. 1), S64-S71. doi: 10.2337/dc12s064

[3] Bengio, Y., and Grandvalet, Y. (2005). Bias in Estimating the Variance of K -Fold Cross-Validation. New York, NY: Springer, 75-95. doi: 10.1007/0-387-24555-3_5

[4] Breiman, L. (2001). Random forest. Mach. Learn. 45, 5-32. doi: 10.1023/A:1010933404324

[5] Chen, X. X., Tang, H., Li, W. C., Wu, H., Chen, W., Ding, H., et al. (2016). Identification of bacterial cell wall lyases via pseudo amino acid composition. Biomed. Res. Int. 2016:1654623. doi: 10.1155/2016/1654623

[6] Cox, M. E., and Edelman, D. (2009). Tests for screening and diagnosis of type 2 diabetes. Clin. Diabetes 27, 132-138. doi: 10.2337/diaclin.27.4.132

[7] Duygu,ç., and Esin, D. (2011). An automatic diabetes diagnosis system based on LDA-wavelet support vector machine classifier. Expert Syst. Appl. 38, 8311-8315.

[8] Friedl, M. A., and Brodley, C. E. (1997). Decision tree classification of land cover from remotely sensed data. Remote Sens. Environ. 61, 399409.

[9] Georga, E. I., Protopappas, V. C., Ardigo, D., Marina, M., Zavaroni, I., Polyzos, D., et al. (2013). Multivariate prediction of subcutaneous glucose concentration in type 1 diabetes patients based on support vector regression. IEEE J. Biomed. Health Inform. 17, 71-81. doi: 10.1109/TITB.2012.2219876

[10]Habibi, S., Ahmadi, M., and Alizadeh, S. (2015). Type 2 diabetes mellitus screening and risk factors using decision tree: results of data mining. Glob. J. Health Sci. 7, 304-310. doi: 10.5539/gjhs.v7n5p304

[11]Han, L., Luo, S., Yu, J., Pan, L., and Chen, S. (2015). Rule extraction from support vector machines using ensemble learning approach: an application for diagnosis of diabetes. IEEE J. Biomed. Health Inform. 19, 728-734. doi: 10.1109/JBHI.2014.2325615

[12]Iancu, I., Mota, M., and Iancu, E. (2008). "Method for the analysing of blood glucose dynamics in diabetes mellitus patients," in Proceedings of the 2008 IEEE International Conference on Automation, Quality and Testing, Robotics, Cluj-Napoca. doi: 10.1109/AQTR.2008.4588883 
Vol. 10, Issue 12, December 2021

\section{DOI: $10.17148 /$ IJARCCE.2021.101209}

[13]Jackson, D. A. (1993). Stopping rules in principal components analysis: a comparison of heuristical and statistical approaches. Ecology 74, $2204-$ 2214. doi: $10.2307 / 1939574$

[14]Jegan, C. (2014). Classification of diabetes disease using support vector machine. Microcomput. Dev. 3, 1797-1801.

[15]Jia, C., Zuo, Y., and Zou, Q. (2018). O-GlcNAcPRED-II: an integrated classification algorithm for identifying O-GlcNAcylation sites based on fuzzy undersampling and a K-means PCA oversampling technique. Bioinformatics 34, 2029-2036. doi: 10.1093/bioinformatics/bty039

[16]Jiang, Y., and Zhou, Z. H. (2004). Editing training data for kNN classifiers with neural network ensemble. Lect. Notes Comput. Sci. 3173, 356361. doi: 10.1007/978-3-540-28647-9_60

[17]Jolliffe, I. T. (1998). "Principal components analysis," in Proceedings of the International Conference on Document Analysis and Recognition (Heidelberg: Springer). 